\title{
CD4 Lymphocytes Decreased
}

National Cancer Institute

\section{Source}

National Cancer Institute. CD4 Lymphocytes Decreased. NCI Thesaurus. Code C78233.

A laboratory test result which indicates decreased levels of CD4 lymphocytes in a biospecimen. 